\title{
Point-of-Care Ultrasound: New Concepts and Future Trends
}

\author{
Yaoting Wang \\ Bengbu Medical College \\ Huihui Chai \\ Bengbu Medical College \\ Ruizhong Ye \\ Hangzhou Medical College \\ Jingzhi Li \\ Beijing Xuanwu Hospital \\ Ji-Bin Liu \\ Thomas Jefferson University \\ Follow this and additional works at: https://jdc.jefferson.edu/radiologyfp \\ See next page for additional authors \\ Part of the Radiology Commons \\ Let us know how access to this document benefits you
}

\section{Recommended Citation}

Wang, Yaoting; Chai, Huihui; Ye, Ruizhong; Li, Jingzhi; Liu, Ji-Bin; Lin, Chen; and Peng, Chengzhong, "Point-of-Care Ultrasound: New Concepts and Future Trends" (2021). Department of Radiology Faculty Papers. Paper 117.

https://jdc.jefferson.edu/radiologyfp/117

This Article is brought to you for free and open access by the Jefferson Digital Commons. The Jefferson Digital Commons is a service of Thomas Jefferson University's Center for Teaching and Learning (CTL). The Commons is a showcase for Jefferson books and journals, peer-reviewed scholarly publications, unique historical collections from the University archives, and teaching tools. The Jefferson Digital Commons allows researchers and interested readers anywhere in the world to learn about and keep up to date with Jefferson scholarship. This article has been accepted for inclusion in Department of Radiology Faculty Papers by an authorized administrator of the Jefferson Digital Commons. For more information, please contact: JeffersonDigitalCommons@jefferson.edu. 


\section{Authors}

Yaoting Wang, Huihui Chai, Ruizhong Ye, Jingzhi Li, Ji-Bin Liu, Chen Lin, and Chengzhong Peng 


\title{
Point-of-Care Ultrasound: New Concepts and Future Trends
}

\author{
Yaoting Wang, MD ${ }^{a, 1}$, Huihui Chai, MD ${ }^{a, 1}$, Ruizhong Ye, MD ${ }^{b}$, Jingzhi Li, MD, PhD ${ }^{c}$, Ji-Bin Liu, MD ${ }^{d}$, Chen Lin ${ }^{a}$, \\ Chengzhong Peng, $\mathrm{MD}^{a, b \text {, * }}$
}

${ }^{a}$ Graduate Department, Bengbu Medical College, Bengbu, Anhui, China; ${ }^{b}$ Department of Ultrasound Medicine, Zhejiang Provincial People's Hospital, Affiliated People's Hospital, Hangzhou Medical College, Hangzhou, Zhejiang, China; ${ }^{c}$ Department of Vascular Ultrasound, Beijing Xuanwu Hospital, Capital Medical University, Beijing, China; ${ }^{d}$ Department of Radiology, Thomas Jefferson University, Philadelphia, PA, USA

Received August 09, 2021; revision received August 28, 2021; accepted August 30, 2021

\begin{abstract}
Ultrasound (US) technology, with major advances and new developments, has become an essential and first-line imaging modality for clinical diagnosis and interventional treatment. US imaging has evolved from one-dimensional, twodimensional to three-dimensional display, and from static to real-time imaging, as well as from structural to functional imaging. Based on its portability and advanced digital imaging technique, US was first adopted by emergency medicine in the 1980s and gradually gained popularity among other specialists for clinical diagnosis and interventional treatment. Point-of-Care Ultrasound (POCUS) was then proposed as a new concept and developed for new uses, which greatly extended clinical US applications. Nowadays, artificial intelligence (AI), cloud computing, 5G network, robotics, and remote technologies are starting to be integrated into US equipment. US systems have gradually evolved to an intelligent terminal platform with powerful imaging and communication tools. In addition, specialized US machines tend to be more suitable and important to meet increasing demands and requirements by various clinical specialties and departments. In this article, we review current US technology and POCUS as new concepts and its future trends, as well as related technological developments and clinical applications.
\end{abstract}

Key words: Ultrasound; Point-of-care ultrasound; Specialty ultrasound; Artificial intelligence; 5G network; Application

Advanced Ultrasound in Diagnosis and Therapy 2021; 03: 268-276

DOI: 10.37015/AUDT.2021.210023

$\mathrm{M}$ odern medical ultrasound (US) technology has made tremendous advancements over the years with many breakthroughs. These revolutionary milestones include developing digital high resolution grayscale US for anatomic structure imaging display, establishing color and power Doppler flow imaging for cardiovascular functional evaluation, creating quantitative elastographic imaging for tissue stiffness measurement, and inventing contrast-enhanced US technology for the integration of structural and functional imaging [1]. With technological advancements and miniaturization of US systems, portable US emerged and has developed since the 1980s, first used by emergency medicine physicians [2] and then extended to other clinical specialists. Clinicians in various disciplines have gradually applied US technology to the diagnosis and treatment of their respective specialties, using the imaging advantages of US technology to implement focused, purposeful, and rapid US examinations to provide clinical information for making diagnoses and treatment decisions, namely Point-of-Care Ultrasound (POCUS) [2]. With the evolvement of artificial intelligence (AI), cloud computing, 5G network, robototics, and remote technology, as well as increasing

\footnotetext{
${ }^{1}$ These two authors contributed equally to the article and should be considered as co-first authors.

* Corresponding author: Graduate Department, Bengbu Medical College, No. 2600, Donghai Avenue, Bengbu, Anhui, 233030, China. Department of Ultrasound Medicine, Zhejiang Provincial People's Hospital, Affiliated People's Hospital, Hangzhou Medical College, No. 158, Shangtang Road, Hangzhou, Zhejiang, 310014, China.

e-mail:pcz001@126.com
}

2576-2508/@ AUDT 2021 • http://www.AUDT.org

This is an open-access article distributed under the terms of the Creative Commons Attribution 4.0 International license, which permits unrestricted use, distribution and reproduction in any medium provided that the original work is properly attributed. 
demands by clinical specialties requiring more refined clinical diagnosis and treatment brought by precision medicine, the miniaturized, intelligent, and specialized US has become a trend for future development [3].

This article will review traditional US and POCUS as new concepts with a focus on related current US technology and its future trends for clinical application. The value of modern technology in promoting technology improvement and expanding clinical application will be discussed.

\section{Traditional Medical Ultrasound}

Medical US is a safe, simple, real-time, easily operated, low cost, and widely used imaging modality in medicine besides CT and MRI [4]. Since the 1940s, medical US technology has evolved from A-mode, M-mode, B-mode (2D gray scale) to Doppler-mode imaging. B-mode US developed in the 1950 s could display the soft tissue structures, which laid the foundation of modern US diagnosis [5]. Subsequently, M-mode US imaging was formed with integration of tissue movement information (such as cardiac motion) [6]. In the mid to late $1980 \mathrm{~s}$, color Doppler US was developed based on the principle of Doppler frequency shift and auto correlation is used to obtain blood flow information, which is coded in different colors. Doppler spectrum analysis can measure the velocity of blood flow and obtain the hemodynamic information over time, which is a spectral Doppler technique. The establishment of Doppler US technology has created a new field for non-invasive testing of cardiovascular and organ blood flow and hemodynamic research $[7,8]$. Up to now, the above several imaging methods are still the mainstream technologies in medical US. With improvement of computer processing power, Baum and Greenwood proposed the original concept of three-dimensional US in 1961[9]. Subsequently, the development of 3D US underwent three stages, which are early static 3D imaging (static 2D image reconstruction), dynamic 3D imaging (adding time parameters) and real-time 3D imaging (with no time delay) [10]. 3D US has been widely used in cardiology, obstetrics, gynecology, oncology, interventional radiology, peripheral vascular, and other fields because of its intuitive, accurate and real-time features [11]. Since the 1990s, new US technologies, including contrast-enhanced US (CEUS) [12], tissue Doppler [13], and elastography [14], have further improved US capabilities and diagnostic accuracy (Fig. 1). In particular, development of microbubble contrast agents for contrast US imaging is a revolutionary breakthrough technique, which played a substantial role in the field of medical imaging. Quantitative analysis of CEUS can be carried out to evaluate the microcirculation perfusion of organs or tissues, which transits the ultrasonic imaging from structure to function [15]. In addition, enhanced US imaging through non-vascular approaches (bile duct, urinary tract, oral administration, etc.) has gradually been applied in clinical practice as well [16-18]. Through the fusion of US technology with other imaging technologies (such as CT and MRI), the ability of lesion detection has also been improved significantly, which improves the lesion localization and interventional operation $[19,20]$.

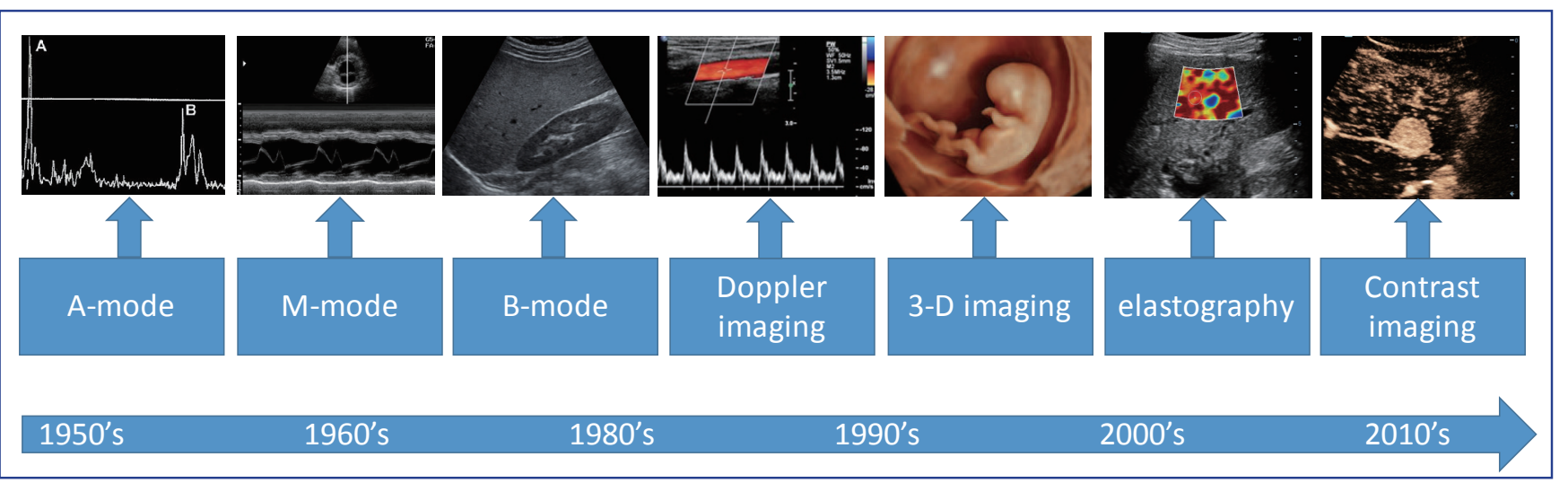

Figure 1 Schematic diagram of US technology developments

After decades of development, medical US has achieved rapid development in terms of imaging technology, imaging modes, software, and hardware. However, traditional US has great challenges in meeting the clinical demand for diagnosis and treatment because of the large size of the equipment, the configuration of the probe, and the imaging features. Although continuous upgrades have been made in probe materials, imaging analysis software, and overall machine design, traditional US technique is relatively mature and has become an established modality. At the same time, due to the lack of equipment/personnel and heavy workload in US 
departments as well as increasing use US by clinical physicians, continuous advancement of US applications has led to the rapid development of POCUS with clinicians/specialties as the main body of users.

\section{Point-of-Care Ultrasound}

Portable B-mode US devices provide the possibility for clinicians to perform bedside real-time US diagnoses. In the 1970s, diagnostic US was first used in the evaluation of trauma patients in Europe. Since 1988, Germany has required surgeons to master US skills. The United States began to promote US assessment of trauma in the mid1980 s, replacing diagnostic abdominal lavage in most trauma centers. Since the 1990s, US has been widely used in acute and critical patients worldwide. Because bedside US emphasizes timeliness and accuracy, the concept of POCUS is proposed in clinical practice $[2,21,22]$.

In 2011, the New England Journal of Medicine described POCUS as "US performed by a clinician at the bedside," emphasizing the "instant" operation by clinicians rather than by professional US physicians [23]. The specific meaning of POCUS is that the clinician is the one who makes the decision to perform bedside US, adjusts the diagnosis, and monitors the treatment accordingly. Based on the advantages of US technology, as well as increasing miniaturization of US instruments and continuous improvement of their functions, POCUS can better meet the clinical needs for different applications. At the same time, with continuous improvement of clinicians' ability to use US, the scope of POCUS application has been greatly expanded, including in the areas of acute and critical care, anesthesia, rehabilitation, rheumatology, obstetrics and gynecology, pain management, sports medicine, and orthopedics, etc. $[24,25]$. In addition, POCUS has played an important role in the fight against the COVID-19 during the pandemic. It can not only make the diagnosis, assess severity, and provide dynamic monitoring of lung lesions, but also provide important information of multi-organ injury (such as heart, kidney, blood vessel, gastrointestinal, etc.). POCUS has helped in classifying cases based on severity and directing patients to appropriate treatment, which greatly improved the therapeutic efficiency [26,27].

The expansion in applications of POCUS are the result of a convergence of advancement of US technology and clinical needs. Because of its advantages in imaging visualization, it is also called the "visual stethoscope" $[28,29]$. The scope of POCUS has increasingly expanded and includes the following applications and scenarios.

\section{Acute and critical care applications}

POCUS can be used in special medical scenarios such as battlefields, first aid, traffic accidents, and disaster scenes. Through rapid assessment of organ damage, it can guide the risk of classification, triage, and treatment measures [30]. Focused assessment with sonography for trauma (FAST) protocol is a bedside examination method for rapid assessment of internal bleeding in trauma patients, with specificity of $94-98 \%$, sensitivity of $73-99 \%$, and accuracy of $90-98 \%$. Also, POCUS is superior to traditional chest radiographs in the diagnosis of pneumothorax [23,31]. The application of POCUS in acute and critical cases pays more attention to volume management and hemodynamics monitoring through streamlined US workflows, such as focused echocardiographic evaluation in life support (FEEL) protocol, bedside lung US in emergency (BLUE) protocol, and rapid US in shock (RUSH) protocol, etc., which can quickly and effectively integrate and interpret critical pathophysiology with US findings, and then guide clinical diagnosis and treatment [32-34].

\section{US-guided visual procedure applications}

When US-guided nerve block (such as subarachnoid space, axillary brachial plexus, sciatic nerve, etc.), the nerve structure and peripheral blood vessels, muscles, bones, and internal organs can be clearly displayed, and the puncture process and drug diffusion can be monitored in real time. Under US-guidance, the blindness of the interventional procedures can be avoided, the success rate of anesthesia can be improved, and the complications and the amount of local anesthesia can be reduced. The integration of systemic US imaging into the clinical practice of anesthesia is a new concept and research direction in the field of POCUS. In addition, US is very useful to guide the establishment of vascular access during a variety of procedures. In the case of patients with poor vascular conditions (such as drug abuse, burns, vascular congenital variability, children, and severe obesity), the advantages of US visualization can improve the precision of venous catheterization and reduce the failure of procedures [35-37].

\section{Musculoskeletal and others applications}

POCUS can be used in rheumatology, rehabilitation, physiotherapy, sports medicine, and orthopedics for dynamic assessment of joint and tendon abnormalities, monitoring of inflammatory lesions, and diagnosis and localization of the cause of pain $[38,39]$. In terms of basic obstetric POCUS examination, through standardized training, POCUS can be used by relevant medical staff in underdeveloped countries or remote areas of the world to perform basic examinations of fetal anatomy, umbilical blood flow status, and fetal position, gestational age, and fetus growth and maternal labor process which could effectively reduce maternal and fetal mortality $[38,40]$. 
In order to meet the above-mentioned clinical applications, equipment manufacturers are also constantly modifying the US instruments to provide better systems for POCUS needs, such as: (1) Removing non-essential function and parameter control buttons, or customizing function keys, simplify operating steps and making the operation faster and more user-friendly; (2) Shortening the switching speed of the ultrasonic instrument, improving the operation response, increasing probe switching and storage speed to meet the needs of emergency scanning; (3) Making more compact equipment with portable designs accustomed to the limited work space in the emergency room, anesthesia suite and intensive care unit as well as other small space environments $[41,42]$.

POCUS is a one-step forward examination method compared to routine clinical US. In the POCUS inspection process, important information such as organ anatomy, functional status, and systemic hemodynamics can be obtained in-situ and in a timely fashion, helping clinicians to make more accurate assessments for immediate and subsequent treatment. POCUS can provide reliable essential information, and participate in the entire clinical diagnosis and treatment process (i.e., preliminary diagnosis, initial treatment, efficacy evaluation, and adjustment of therapy plan). Thus, the application of POCUS for multi-disciplinary, multisystem, and multi-organ assessment is a future direction and a key link in various clinical disciplines with broad clinical application prospects $[43,44]$.

\section{POCUS with New Concept and Techniques}

In recent years, POCUS has been applied in many clinical departments and achieved encouraging results. However, in most hospitals at present, portal US is still performed by US doctors/radiologists with bedside consultations. In addition to being somehow unfamiliar with the purpose of clinical specialist's requests, it is also impossible to achieve continuous follow-up observation for special patients, such as FAST examination for trauma patients [45] and cardiopulmonary US for patients with ECMO treatment $[46,47]$. Some clinical departments in large-scale hospitals have been equipped with portable US and carry out POCUS examinations. However, the lack of effective operating standardization, high scanning skill, and interpretation experience leads to ambiguous diagnosis results, which diminishes the clinical application value of POCUS [48].

Although portable US equipment has achieved technical integration (i.e., multiple imaging functions in a single unit) and miniaturization (such as handheld US device), there are still challenges for development of US devices and applications to move forward into a subspecialty of clinical disciplines. For example, in anesthesiologic uses, clearly showing the boundary of normal tissue structure is more important than the diagnosis of pathological structures. A touch screen that is easier to sterilize is better than the traditional operating keyboard [49]. Therefore, a specially-designed US machine with new concepts is needed in developing specialty-oriented instruments.

With the rapid integration of modern technologies such as artificial intelligence (AI), cloud computing, $5 \mathrm{G}$ networks, robots, and tele-remote technology with US modality, the development of a specialized POCUS system will be further facilitated as an intelligence terminal platform, improving the application of POCUS and leading to a new pathway for medical US advancement (Fig. 2). New generations of POCUS systems will represent a development trend of medical US. In addition to using a variety of new technologies to improve the accuracy of US diagnosis, speciallydesigned POCUS will be combined with advanced technology to better meet the needs of various specialists which may differ from the traditional US systems.

\section{G-based tele-remote POCUS}

Tele-remote US refers to using modern computer, network communication, and multimedia technology to digitally reconstruct US images to remotely achieve image acquisition, storage, transmission, analysis, and processing. This allows for remote real-time diagnosis and interventional procedures through high-precision synchronization via video, audio, text and other multichannel communications. Expert doctors can use remote technology to guide patient-side doctors to perform US examinations to improve their diagnostic experience, and reduce diagnostic errors by overcoming operatordependent barrier. The remote robotic US system enables expert doctors to use their own skill to remotely control the robot to perform US scans, and perform medical diagnosis based on real-time US imaging generated by the robotic scanning [50]. In recent years, emerging $5 \mathrm{G}$ technology has met the long-distance, real-time, high-bandwidth, high-resolution, and low-latency requirements for remote US consultation and US robotic operation, allowing for high-quality transmission of US imaging and sharing as a valuable medical resource, which provides the opportunity for broad application of tele-remote US technology. The 5G-based remote US has shown important value during the COVID-19 pandemic for remote assessment of patients' lung lesions and guidance during interventional procedures, saving expert resources and minimizing cross-infection [50,51]. However, remote US is not conducted for large-scale 
clinical application, and it can only be used as a basic screening tool for special situations at present due to the lack of unified standards for image acquisition, quality control, data transmission, and security $[52,53]$.

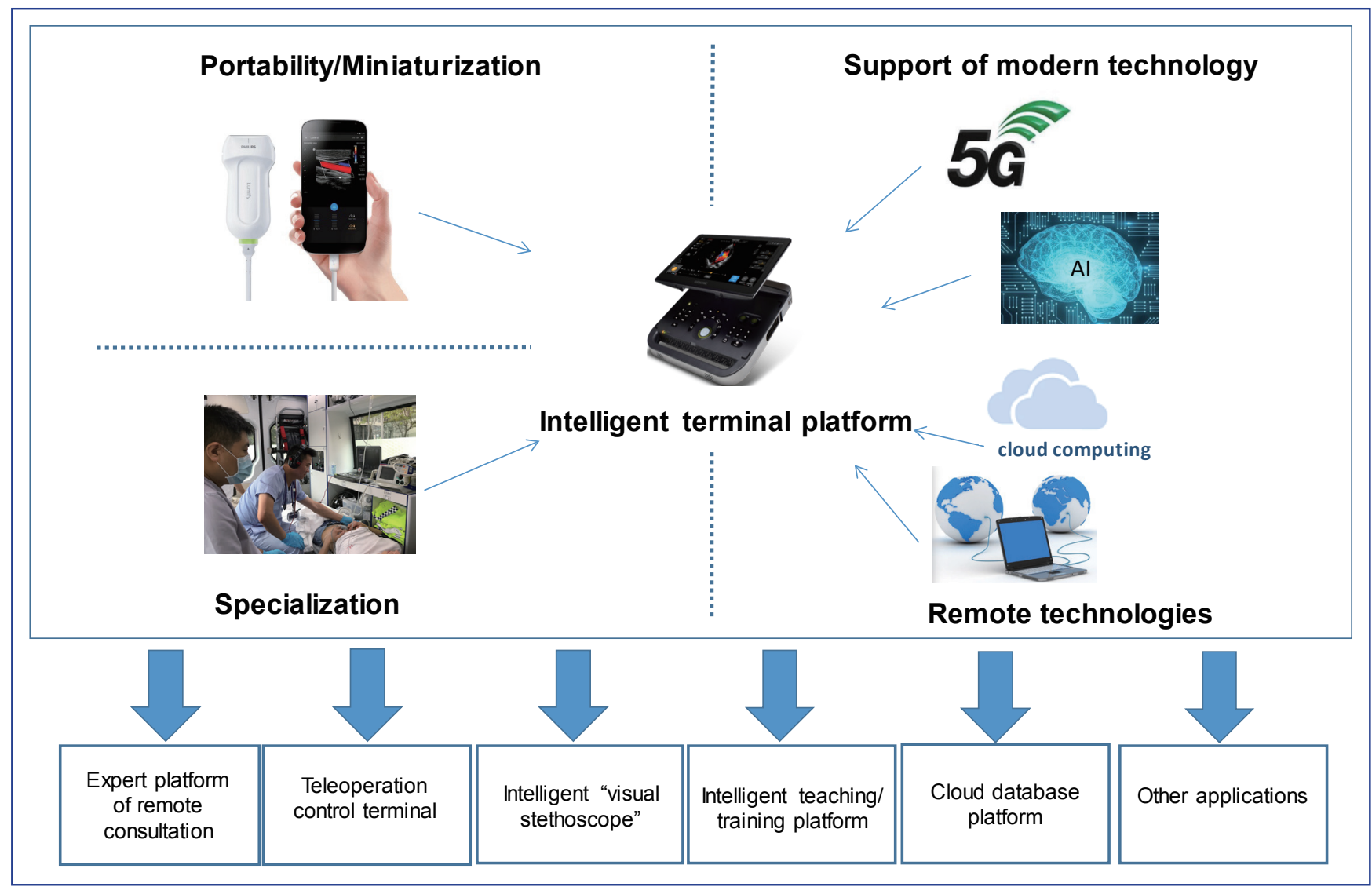

Figure 2 Conception of ultrasound intelligent terminal platform

\section{POCUS with AI technology}

Sonologists/radiologists who independently perform US diagnoses often require years of training and experience accumulation. Currently, diagnostic US is the first choice for clinical imaging examination, and as a result, the demand for diagnostic US scans can be overwhelmed by a heavy workload with limited staffing. With AI technology being gradually implemented in the medical field, AI-assisted diagnosis systems could help alleviate this situation although applying AI in diagnostic US is a relatively new concept in the field of digital imaging. Advantages of AI include fast imaging processing, uniform standards, continuous workflow, and excellent repeatability, which can handle large amounts of data and quickly obtain diagnostic images and even dig out the raw data and patterns to improve diagnostic efficiency and accuracy. In addition, through the continuous optimization or iterative update of AI algorithms, US imaging processing and analysis can be simultaneously improved, and ultimately realize intelligent disease prediction, risk assessment, clinical diagnosis, and treatment $[54,55]$.
Deep learning methods are commonly used in AI technologies, in which convolutional neural network $(\mathrm{CNN})$ is the most popular, and has made great progress in various research areas such as image classification, lesion detection, and target segmentation [56,57]. Buda et al. [58] used CNN to develop an intelligent recommendation algorithm for whether thyroid lesions need biopsy, with a sensitivity of $87 \%$ and a specificity of $52 \%$. CNN is composed of many convolutional layers which have the powerful ability to independently learn a large amount of imported image training data, and automatically parse and extract the best characteristics in a specific layer, and finally, realize the classification of the image. This process is much better with more detailed information than human eye observation which is the biggest advantage of CNN compared with traditional machine learning methods. Traditional machine learning uses artificially defined characteristics, such as whether the edges of the nodules are regular and the echogenicity, based on the doctor's subjective judgment criteria [59].

US equipment has also made great progress with AI technology, such as the built-in intelligent evaluation 
features, intelligent optimization of imaging quality and intelligent screening, acquisition, analysis, and data processing, etc., helping US operators to bypass the complicated image optimization and measurement work, and instead focus on clinical diagnosis and treatment. At present, US with AI technology has been applied in clinical practice such as minimally invasive intervention, thyroid, breast, musculoskeletal, pediatrics, and cardiac examinations, improving the accuracy of clinical US diagnosis [60-65]. For example, the coincidence rate by AI-based US systems in the interpretation of benign and malignant thyroid nodules has increased from $64 \%$ to $84 \%$ [66]. However, there are still many challenges in AI US applications. First, the huge quantity of data generated in the short term puts higher requirements on algorithms and computing power. Second, the computing power limitation needs to be solved to ensure that the AI model can be effectively used on tablets or mobile phone platforms [67]. Finally, it is necessary to establish supervision systems and regulations and improve the scheme to evaluate the stability and accuracy and overcome the difficulty of defining medical liability for AI US applications.

\section{Cloud-based POCUS}

The rapid development of the Internet brings up cloud computing applications. In 2006, Amazon in the United States launched the world's first cloud computing system-Amazon Web Services (AWS), and cloud computing began to gain rapid promotion globally. Cloud computing is a new type of computing platform that has the advantages of low cost, high reusability, high performance, and easy expansion. Through the internet, it accelerates the integration of a large number of algorithm formulas and storage resources, and then provides and distributes to specific users accordingly. Currently, the definition given by the National Institute of Standards and Technology (NIST) in the United States is more authoritative for implantation. The main goal of cloud computing is to form a pool of resources for network, storage, application, service, and other resources, and strengthen flexible allocation and integration, as well as optimize network resources. Through these approaches, cloud computing can enhance its reliability, versatility, and expansibility to maximize the utilization of network information resources [68].

US technology has been widely used in the diagnosis and treatment of diseases in almost all fields of medicine. Thus, a large volume of imaging data is generated due to the characteristics of real-time imaging. Using cloud computing technology to build a cloud platform for medical image service will make data processing simple and convenient. Recently, with the application of mobile terminal devices such as mobile phones or tablet computers, cloud computing technology has brought new changes for US diagnosis. The US system on the patient side is responsible for collecting image data, while the mobile device on the doctor side displays the image data. Through 5G technology and cloud platforms, the real-time transmission of image data between the two locations and the implementation of remote consultation can be realized [69]. The cloud service platform can also endow users with huge storage capacity and high reliability. After US equipment is integrated into a cloud computing system, it could effectively increase the processing speed, optimize the allocation of resources, realize the interconnection and intercommunication between various terminal devices, and become a veritable "cloud US", enabling instant sharing of medical information, resources, and services [70]. Since "cloud US" is a new concept, it is necessary to further develop industry standards and technology for its potential applications.

\section{Specialty-oriented POCUS}

The configuration of transducers (such as shape and frequency) and machines for whole-body high-end US systems often have a universal design and lack of specific consideration for clinical specialty uses. The key diagnostic information of the heart, hemodynamics, and lungs has to be obtained through complex operations or measurements by experienced sonologists. Specialty clinicians without an indepth understanding of US principles and operating training may not be able to operate sophisticated US devices for their point-of-care applications. Thus, development of the specially-configurated US instruments is clearly necessary for clinical specialties to be used [71,72]. For example, the anesthesiologist requires US scanner to have a more refined ability to recognize superficial nerve structures and to have the function of puncture navigation, while the emergency and critical care physician needs US system to be rapidly activated and intuitive operation; a musculoskeletal specialist needs US unit to have better imaging resolution for visualization of superficial tissues (Fig. 3).

With POCUS development, specialized US products that meet various specialty needs for clinical application have been developed by many US companies such as portable US machines by GE $\left(\right.$ Venue $\left.^{\mathrm{TM}}\right)$, Mindray $\left(\mathrm{M} 9^{\mathrm{TM}}\right)$, Sonoscape $\left(\mathrm{S} 9^{\mathrm{TM}}\right)$, Wisonic $\left(\mathrm{Navi}^{\mathrm{TM}}\right)$, and hand-hold US scanners by Philips (Lumify $\left.{ }^{\mathrm{TM}}\right)$, Butterfly $\left(\mathrm{iQ}^{+{ }^{\mathrm{TM}}}\right)$, Chison (SonoEye V5 ${ }^{\mathrm{TM}}$ ), Stork $\left(\mathrm{S} 35^{\mathrm{TM}}\right.$ ), SonoStar $\left(\right.$ Bprobe $^{\mathrm{TM}}$ ) and so on (Fig. 4). For example, GE Venue ${ }^{\mathrm{TM}}$ is specifically developed for use by clinicians in the field of emergency and critical care, and is dedicated for specialty needs, including intelligent functional design (such as the intelligent BLUE and RUSH protocols based on deep learning technology and VTI intelligent 


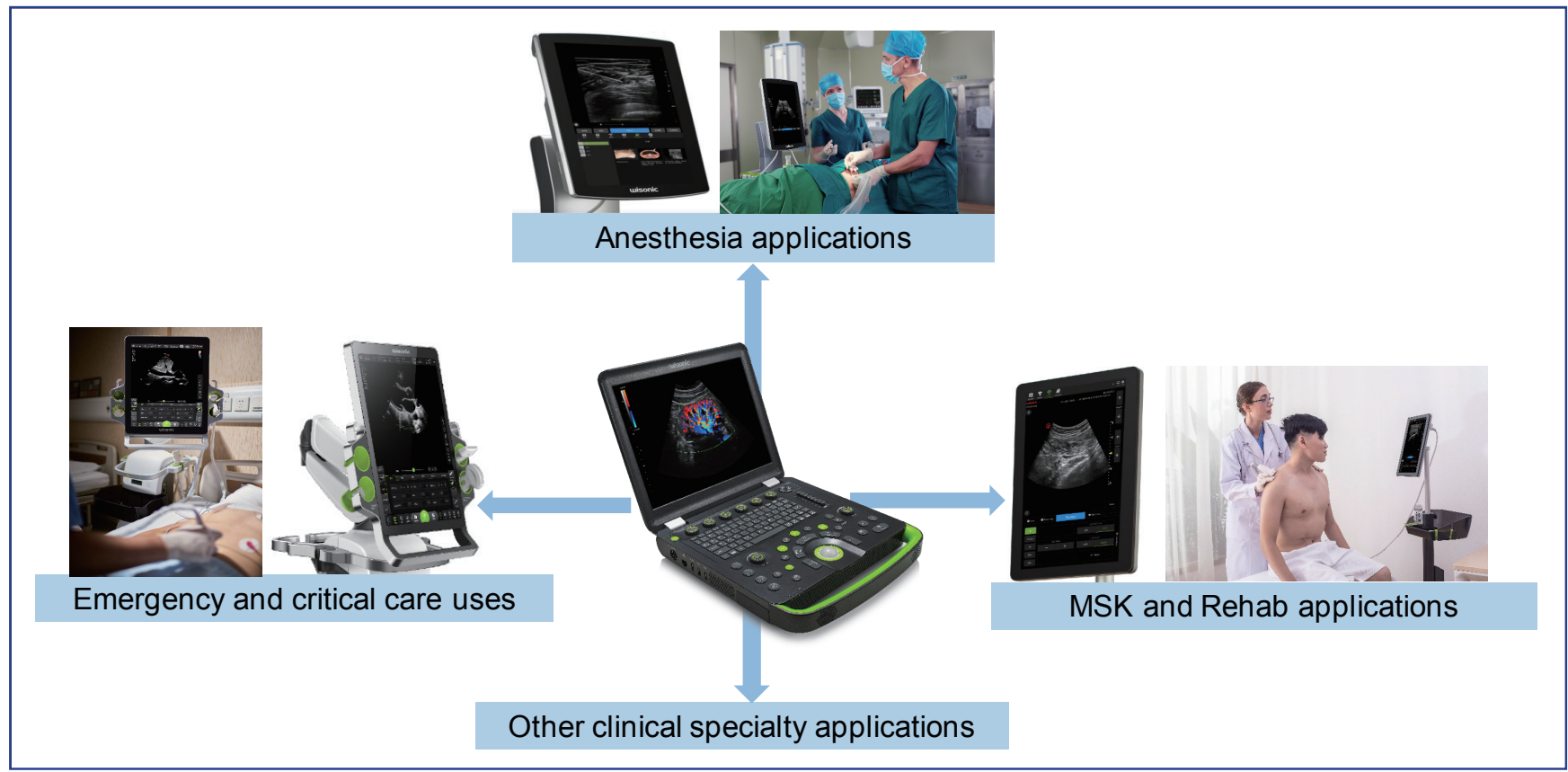

Figure 3 Illustration of portable US systems for clinical specialty applications

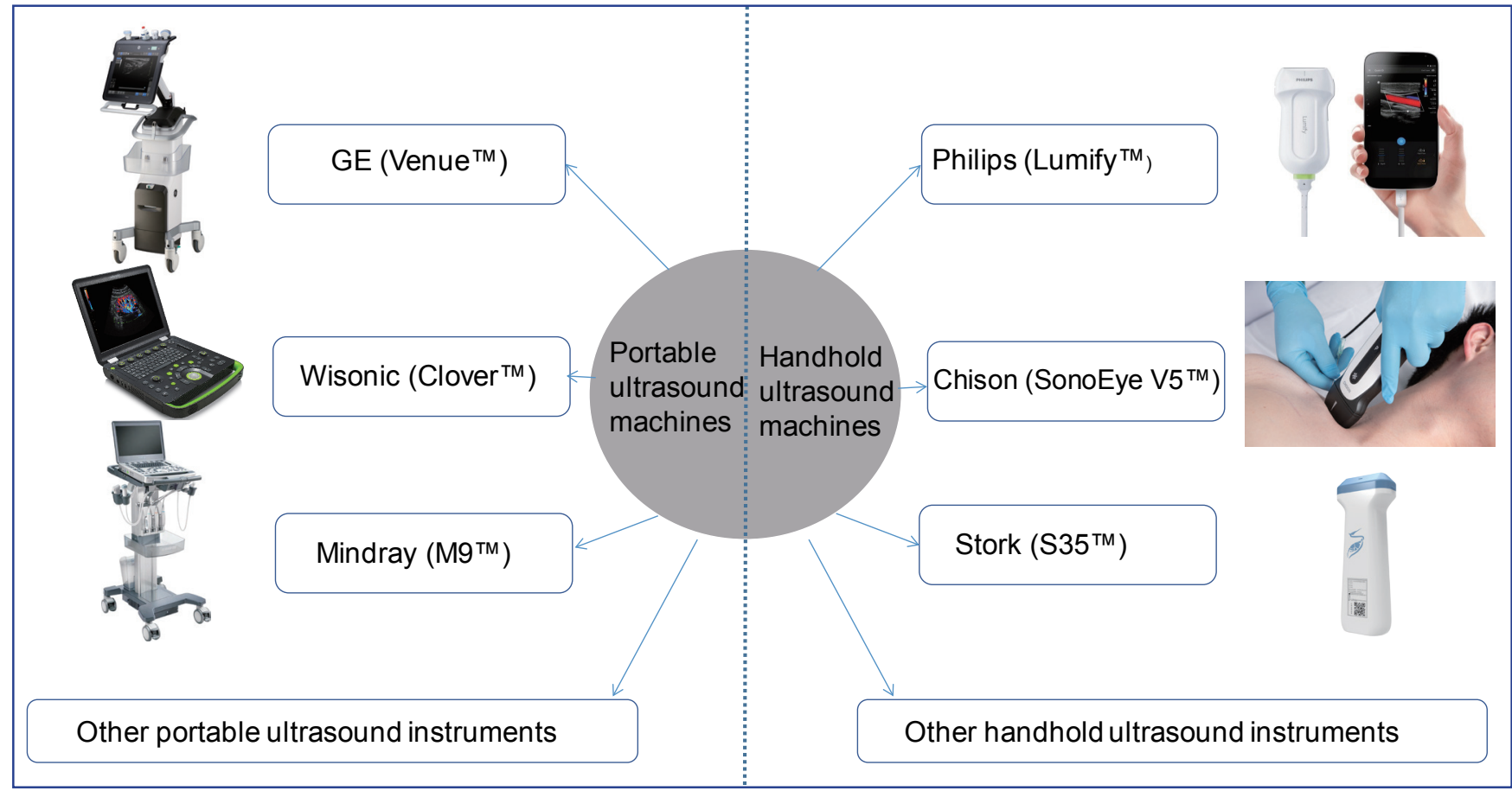

Figure 4 Examples of portable and handheld ultrasonic instruments

measurement) with simple and efficient operation process $[72,73]$. Wisonic $\mathrm{Navi}^{\mathrm{TM}}$ is an all-in-one machine specifically designed for anesthesiologic applications and equipped with a large touch screen for far-distance observation and easy disinfection. Customizable functional buttons are built on the probe for Doppler control and parameter adjustment as well as mode switching and needle enhancement control. Thus, POCUS devices must fit with specific applications and requirements by specialty clinicians, which is expected to become a key development area of US systems in the future. In the meantime, multiple specialized US techniques can also be used in the same clinical scenario to carry out multi-organ or multi-modal US evaluation [33].

\section{Conclusion}

POCUS, endowed with new concepts and prospects, 
will greatly extend clinical US applications. Currently, AI, cloud computing, $5 \mathrm{G}$ network, robotics, and remote technologies started to integrate into US equipment. US systems have gradually evolved to an intelligent terminal platform with powerful imaging functions. In addition, customized US machines tend to be more suitable and are important to meeting the increasing requirements by various clinical specialties and departments. POCUS not only represents a modern technology revolution but also expands scenarios of US applications.

\section{Acknowledgment}

This work was supported by the General Research Project of Department of Education of Zhejiang Province (Grant number: Y202044583), Zhejiang Medicine Scientific and Technology Project (Grant Number: 2021KY026).

\section{Conflicts of interest}

The authors declare no conflicts of interest.

\section{References}

[1] Dong BW. The third revolution of modern ultrasound imaging: realtime contrast-enhanced ultrasound technology (outline). Hainan: Proceedings on New Advances in Ultrasound Medicine of the Chinese Medical Association Conference, 2004: 29-32.

[2] Li L. Emergency ultrasound-based on today, looking forward to the future. Chinese Journal of Emergency Medicine 2020;29: 8-11.

[3] Liu Y, Lv F, Li T. The 5G ultrasound era is coming: the current status and progress of remote ultrasound applications. Chinese Journal of Medical Ultrasound (Electronic Edition) 2019; 16: 241-243.

[4] Wang DC, Jang HJ, Kim TK. Characterization of indeterminate liver lesions on CT and MRI with contrast-enhanced ultrasound: What is the evidence? AM J Roentgenol 2020; 214: 1295-1304.

[5] Edler I, Lindström K. The history of echocardiography. Ultrasound Med Biol 2004; 30: 1565-1644.

[6] Bonagura JD, Blissitt KJ. Echocardiography. Equine Vet J Suppl 1995; 19: 5-17.

[7] Kim ES, Sharma AM, Scissons R, Dawson D, Eberhardt RT, Gerhard-Herman M, et al. Interpretation of peripheral arterial and venous Doppler waveforms: a consensus statement from the society for vascular medicine and society for vascular ultrasound. VASC Med 2020; 25: 484-506.

[8] Stevenson JG. The development of color Doppler echocardiography: innovation and collaboration. $J$ Am Soc Echocardiogr 2018; 31: 1344-1352.

[9] Baum G, Greenwood I. Orbital lesion localization by threedimensional ultrasonography. N Y State J Med 1961; 61: 4149-4157.

[10] Adriaanse BM, van Vugt JM, Haak MC. Three- and four-dimensional ultrasound in fetal echocardiography: an up-to-date overview. $J$ Perinatol 2016; 36: 685-693.

[11] Goo HW, Park SJ, Yoo SJ. Advanced medical use of threedimensional imaging in congenital heart disease: augmented reality, mixed reality, virtual reality, and three-dimensional printing. Korean J Radiol 2020; 21: 133-145.

[12] Erlichman DB, Weiss A, Koenigsberg M, Stein MW. Contrast enhanced ultrasound: a review of radiology applications. Clin Imaging 2020; 60: 209-215.

[13] Bank AJ, Kelly AS. Tissue Doppler imaging and left ventricular dyssynchrony in heart failure. $J$ Card Fail 2006; 12: 154-162.

[14] Sigrist RMS, Liau J, Kaffas AE, Chammas MC, Willmann JK. Ultrasound elastography: review of techniques and clinical applications. Theranostics 2017; 7: 1303-1329.

[15] Chong WK, Papadopoulou V, Dayton PA. Imaging with ultrasound contrast agents: current status and future. Abdom Radiol (NY) 2018; 43: 762-772.

[16] Wang Y, Yang Y, Wang K, Tang S. The value of contrast-enhanced ultrasound-guided contrast injection via the endoscopic nasobiliary drainage duct in diagnosing residual common bile duct stones. Biomed Res Int 2020: 3281241.

[17] Chua Me, Kim JK, Mendoza JS, Fernandez N, Ming JM, Marson A, et al. Evaluation of vesicoureteral reflux among children using contrasted-enhanced ultrasound: a literature review. J Pediatr Urol 2019; 15: 12-17.

[18] He X, Sun J, Huang X, Zeng C, Ge Y, Zhang J. Comparison of oral contrast-enhanced transabdominal ultrasound imaging with contrastenhanced computed tomography in preoperative tumor staging of advanced gastric carcinoma. J Ultrasound Med 2017; 36: 2485-2493.

[19] Moro F, Gui B, Arciuolo D, Bertoldo V, Borzi R, Romeo P, et al. Fusion imaging of ultrasound and MRI in the assessment of locally advanced cervical cancer: a prospective study. Int $J$ Gynecol Cancer 2020; 30: 456-465.

[20] Xu E, Li K, Long Y, Luo L, Zeng Q, Tan L, et al. Intra-procedural $\mathrm{CT} / \mathrm{MR}$-ultrasound fusion imaging helps to improve outcomes of thermal ablation for hepatocellular carcinoma: results in 502 nodules. Ultraschall Med 2021; 42: e9-e19.

[21] Gleeson T, Blehar D. Point-of-care ultrasound in trauma. Semin Ultrasound CT MR 2018; 39: 374-383.

[22] Dietrich CF, Goudie A, Chiorean L, Cui XW, Gilja Oh, Dong Y, et al Point of care ultrasound: A WFUMB position paper. Ultrasound Med Biol 2017; 43: 49-58.

[23] Moore CL, Copel JA. Point-of-care ultrasonography. $N$ Engl J Med 2011; 364: 749-757.

[24] Soni NJ, LucasBP. Diagnostic Point-of-care ultrasound for hospitalists. J Hosp Med 2015; 10: 120-124.

[25] Liao SF, Chen PJ, Chaou Ch, Lee Ch. Top-cited publications on point-of-care ultrasound: the evolution of research trends. $A m \mathrm{~J}$ Emerg Med 2018; 36: 1429-1438.

[26] Hussain A, Via G, Melniker L, Goffi A, Tavazzi G, Neri L, et al. Multi-organ point-of-care ultrasound for COVID-19 (POCUS4COVID): International Expert Consensus. Crit Care 2020; 24: 702.

[27] Lv F, Wang J, Yu X, Yang A, Liu JB, Qian L, et al. Chinese expert consensus on critical care ultrasound applications at COVID-19 pandemic. Advanced Ultrasound in Diagnosis and Therapy 2020; 4 $27-42$.

[28] Gillman LM, Kirkpatrick AW. Portable bedside ultrasound: The visual stethoscope of the 21st century. Scand J Trauma Resusc Emerg Med 2012; 20: 18.

[29] Varsou O. The use of ultrasound in educational settings: What should we consider when implementing this technique for visualisation of anatomical structures? Adv Exp Med Biol 2019; 1156: 1-11.

[30] Zhou J, Huang J, Wu H, Jiang H, Zhang H, Prasoon P, et al. Screening of ultrasonography of 2,204 patients with blunt abdominal trauma in the Wenchuan earthquake. J Trauma Acute Care Surg 2012; 73: 890-894.

[31] Kirkpatrick AW, Sirois M, Laupland KB, Liu D, Rowan K, Ball CG, et al. Han-held thoracic sonography for detecting post-traumatic pneumothoraces: the extended assessment with sonography for trauma (EFast). J Trauma 2004; 57: 288-295.

[32] Wang XT, Liu DW, Zhang HM, He HW, Liu Y, Chai WZ, et al. The value of bedside lung ultrasound in emergency-plus protocol for the assessment of lung consolidation and atelectasis in critical patients. 
Chinese Journal of Internal Medicine 2012; 51: 948-951

[33] Wang XT, Liu DW, Jiang K, Guan XD, Ma XC, Yan J, et al. The consensus of Chinese Critical Ultrasound Experts. Clinical Focus 2017; 32: 369-383.

[34] Liu DW, Wang XT, Zhang HM, Jiang K, Long Y, Tang YQ, et al. Treatment of severe hemodynamics-Beijing consensus. Chinese Journal of Internal Medicine 2015; 54: 248-271.

[35] Li L, Yong RJ, Kaye AD, Urman Rd. Perioperative point of care ultrasound (Pocus) for anesthesiologists: an overview. Curr Pain Headache Rep 2020; 24: 20.

[36] Pulton D, Feinman J. Hocus POCUS: Making barriers to perioperative point-of-care ultrasound disappear. J Cardiothorac Vasc Anesth 2019; 33: 2419-2420.

[37] Schoch M, Bennett PN, Currey J, Hutchinson AM. Point-of-care ultrasound use for vascular access assessment and cannulation in nutritional status: a scoping review. Semin Dial 2020; 33: 355-368.

[38] Arnold MJ, Jonas CE, Carter RE. Point-of-care ultrasonography. Am Fam Physician 2020; 101: 275-285.

[39] Situ-LaCasse E, Grieger RW, Crabbe S, Waterbrook AL, Friedman L, Adhikari S. Utility of point-of-care musculoskeletal ultrasound in the evaluation of emergency department musculoskeletal pathology. World J Emerg Med 2018; 9: 262-266.

[40] Grytten J, Skau I, Sørensen R, Eskird A. Does the use of diagnostic technology reduce fetal mortality? Health Serv Res 2018; 53: 4437-4459.

[41] Barreiros AP, Dong Y, Ignee A, Wastl D, Dietrich CF. EchoScopy in scanning abdominal diseases; a prospective single center study. Med Ultrason 2019; $21: 8$ to 15 .

[42] Lavi A, Tzemah S, Hussein A, Bishara I, Shcherbakov N, Zelichenko $\mathrm{G}$, et al. A urologic stethoscope? Urologist performed sonography using a pocket-size ultrasound device in the point-of-care setting. Int Urol Nephrol 2017; 49: 1513-1518.

[43] Volpicelli G, Mayo P, Rovida S. Focus on ultrasound in intensive care. Intensive Care Med 2020; 46: 1258-1260.

[44] Neskovic AN, Skinner H, Price S, Via G, De Hert S, Stankovic I, et al. Focus cardiac ultrasound core curriculum and core syllabus of the European Association of Cardiovascular Imaging. Eur Heart $J$ Cardiovasc Imaging 2018; 19: 475-481.

[45] Montoya J, Stawicki SP, Evans DC, Bahner DP, Sparks S, Sharpe RP, et al. From FAST to E-FAST: an overview of the evolution of ultrasound-based traumatic injury assessment. Eur J Trauma Emerg Surg 2016; 42: 119-126.

[46] Douflé G, Roscoe A, Billia F, Fan E. Echocardiography for adult patients supported with extracorporeal membrane oxygenation. Crit Care 2015; 19: 326.

[47] Ntoumenopoulos G, Buscher H, Scott S. Lung ultrasound score as an indicator of dynamic Lung compliance during veno-venous extracorporeal membrane oxygenation. Int J Artif Organs 2021; 44: 194-198.

[48] Manson WC, Kirksey M, Boublik J, Wu CL, Haskins SC. Focused assessment with sonography in trauma (FAST) for the regional anesthesiologist and pain specialist. Reg Anesth Pain Med 2019; 44: 540-548.

[49] Urman RD, Kaye AD. Recent advances in regional anesthesia and ultrasound techniques. Best Pract Res Clin Anaesthesiol 2019; 33: 373-375.

[50] Ye R, Zhou X, Shao F, Xiong L, Hong J, Huang H, et al. The feasibility of a 5G-based robot assisted remote ultrasound system for cardiopulmonary assessment of patients with coronavirus disease 2019. Chest 2021; 159: 270-281.

[51] Lin X, Hu J, Ren L, Huang Y, Wu D, Zhao S, et al. Application of remote ultrasound in COVID-19 isolated intensive care unit. Advanced Ultrasound in Diagnosis and Therapy 2020, 4: 123-127.

[52] Nyeem H, Boles W, Boyd C. A review of medical image watermarking requirements for teradiology. J Digit Imaging 2013;
26: $326-343$.

[53] Choudhri AF, Chatterjee AR, Javan R, Radvany MG, Shih G. Security issues for mobile medical imaging: A primer. Radiographics 2015; 35: 1814-1824.

[54] Cantisani V, Grani G, Tovoli F, Piscaglia F, Catalano C. Artificial intelligence: What is it and how can it expand the ultrasound potential in the future? Ultraschall Med 2020; 41: 356-360.

[55] Muse ED, Topol EJ. Guiding ultrasound image capture with artificial intelligence. Lancet 2020; 396: 749.

[56] Chan HP, Samala RK, Hadjiiski LM, Zhou C. Deep learning in medical image analysis. Adv Exp Med Biol 2020; 1213: 3-21.

[57] Gao J, Jiang Q, Zhou B, Chen D. Convolutional neural networks for computer-aided detection or diagnosis of medical image analysis: an overview. Math Biosci Eng 2019; 16: 6536-6561.

[58] Buda M, Wildman-Tobriner B, Hoang JK, Thayer D, Tessler FN, Leton WD, et al. Management of nodules seen on US images: deep learning may match performance of radiologists. Radiology 2019; 292: 695-701.

[59] Zhou LQ, Wang JY, Yu SY, Wu GG, Wei Q, Deng YB et al. Artificial intelligence in medical imaging of the liver. World $J$ Gastroenterol 2019; 25: 672-682.

[60] Bowness J, El-Boghdadly K, Burckett-St Laurent D. Artificial intelligence for image interpretation in ultrasound-guided regional anaesthesia. Anaesthesia 2021; 76: 602-607.

[61] Wildman-Tobriner B, Buda M, Hoang JK, Middleton WD, Thayer D, Short RG, et al. Using artificial intelligence to revise ACR TI-RADS risk stratification of thyroid nodules: diagnostic accuracy and utility. Radiology 2019; 292: 112-119.

[62] Le EPV, Wang Y, Huang Y, Hickman S, Gilbert FJ. Artificial intelligence in breast imaging. Clin Radiol 2019; 74: 357-366.

[63] Shin Y, Yang J, Lee YH, Kim S. Artificial intelligence in musculoskeletal ultrasound imaging. Ultrasonography 2021; 40: 30-44.

[64] Reismann J, Romualdi A, Kiss N, Minderjahn MI, Kallaracka J, Schad M,et al . Diagnosis and classification of pediatric acute appendicitis by artificial intelligence methods investigatorindependent approach. PloS One 2019; 14: e0222030.

[65] Leeson P, Fletcher AJ. Combining artificial intelligence with human insight to automate echocardiography. Circ Cardiovasc Imaging 2019; 12: e009727.

[66] Ma J, Wu F, Zhu J, Xu D, Kong D. A pre-trained convolutional neural network based on thyroid nodule diagnosis. Ultrasonics 2017 73: 221-230.

[67] Bi WL, Hosny A, Schabath MB, Giger ML, Birkbak NJ, Mehrtash A, et al. Artificial intelligence in cancer imaging: Clinical Challenges and Applications. CA Cancer J Clin 2019; 69: 127-157.

[68] Sobeslav V, Maresova P, Krejcar O, Franca TC, Kuca K. Use of cloud computing in biomedicine. J Biomol Struct Dyn 2016; 34 2688-2697.

[69] Lin CW, Abdul SS, Clinciu DL, Scholl J, Jin X, Lu H et al. Empowering village doctors and enhancing rural healthcare using cloud computing in a rural area of mainland China. Comput Methods Programs BioMed 2014; 113: 585-592.

[70] Hsieh JC, Li AH, Yang CC. Mobile, cloud, and big data computing: Challenges, and new directions in telecardiology. Int $J$ Environ Res Public Health 2013; 10: 6131-6153.

[71] König IR, Fuchs O, Hansen G, von Mutius E, Kopp MV. What is precision medicine? Eur Respir J 2017; 50: 1700391.

[72] Gummadi S, Eisenbrey J, Li J, Li Z, Forsberg F, Lyshchik A, et al Advances in modern clinical ultrasound. Advanced Ultrasound in Diagnosis and Therapy 2018, 2: 51-63.

[73] Bobbia X, Muller L, Claret PG, Vigouroux L, Perez-Martin A, De La Coussaye Je, et al. A new echomapping tool for cardiac output evaluation: an experimental study. Shock 2019; 52: 449-455. 